NISSUNA UMANA INVESTIGAZIONE SI PUO DIMANDARE VERA SCIENZIA S'ESSA NON PASSA PER LE MATEMATICHE DIMOSTRAZIONI LEONARDO DA VINCI

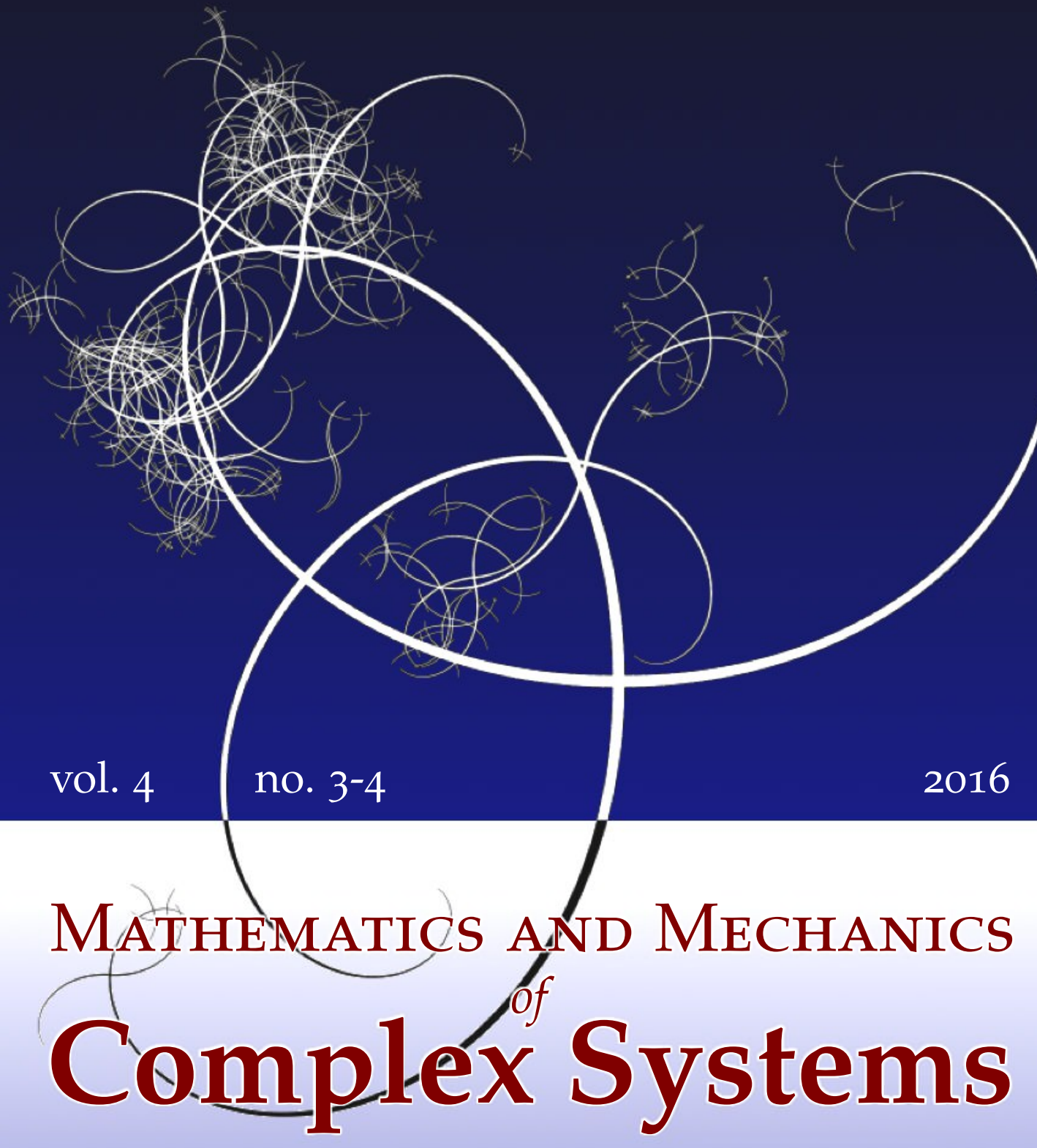

VINCENZO GRECCHI

QUANTUM MECHANICS:

SOME BASIC TECHNIQUES FOR SOME BASIC MODELS II: THE TECHNIQUES 


\title{
QUANTUM MECHANICS: SOME BASIC TECHNIQUES FOR SOME BASIC MODELS II: THE TECHNIQUES
}

\author{
VINCENZO GRECCHI
}

\begin{abstract}
This paper is a continuation of the previous one: "Quantum mechanics: some basic techniques for some basic models, I: The models". The main subject is the perturbation theory in quantum mechanics giving diverging perturbation series for the energy levels of the oscillators. Thus, a short historical introduction on the methods of the sum of diverging power series is given.
\end{abstract}

\section{Introduction and some techniques}

This is the second part of an article in honor of Professor Lucio Russo. In Section 2 there is a short history of the regular methods of the sum of divergent series. It starts with the formal series $\tilde{S}(a)=\sum_{n} a_{n}$, where $a=a_{0}, a_{1}, \ldots$ is considered a vector of components $a_{n}=(-1)^{n}$, with a possible sum $S(a)=\frac{1}{2}$ as suggested by Guido Grandi. The best proof of the value of this sum, if existent, is based on the assumption that $S(a)$ is a linear functional. The same linearity was used by Pietro Mengoli for the sum of some convergent series.

1a. Quadratic estimates. A family of analytic operators of type A should have constant domain [Kato 1966; Reed and Simon 1975; 1978]. For the control on the domain, the quadratic estimates can be used. We consider the simple closed $P T$ operator $H:=O_{3}(0)=p^{2}+i x^{3}$, and we want to prove that $D(H)=D\left(p^{2}\right) \cap D\left(x^{3}\right)$, knowing that $D\left(p^{2}\right) \cap D\left(x^{3}\right) \subset D(H)$. We consider the quadratic norm as a quadratic form

$$
\|H \psi\|^{2}=\left\langle\psi, H^{*} H \psi\right\rangle \quad \text { for } \psi \in D\left(p^{2}\right) \cap D\left(x^{3}\right),
$$

where

$$
H^{*} H=p^{4}+x^{6}+3\left[x^{2}, p\right]_{+}=p^{4}+x^{6}+3\left[\left(p+x^{2}\right)^{2}-p^{2}-x^{4}\right],
$$

\section{Communicated by Francesco dell'Isola.}

PACS2010: 03.65.-w.

Keywords: confinement of the nodes and exact quantization condition, Stokes lines, Stokes sectors, generalized change of representation, Riccati equation and semiclassical series, divergent perturbation series, sum of divergent series. 
and we have the inequality

$$
\begin{aligned}
\|H \psi\|^{2} & \geq\left\|p^{2} \psi\right\|^{2}-3\|p \psi\|^{2}+\left\|x^{3} \psi\right\|^{2}-3\left\|x^{2} \psi\right\|^{2} \\
& >(1-\epsilon)\left(\left\|p^{2} \psi\right\|^{2}+\left\|x^{3} \psi\right\|^{2}\right)-\frac{C}{\epsilon}\|\psi\|^{2}, \quad \epsilon>0,(1-\epsilon)>0, C>0,
\end{aligned}
$$

so that $D(H)$ cannot be extended and $D(H)=D\left(p^{2}\right) \cap D\left(x^{3}\right)$.

1b. The method of Loeffel and Martin for the confinement of the zeros. We explain the Loeffel-Martin method [Loeffel et al. 1969] with a useful example. We consider a positive level $E=\hat{E}_{m}(0)$ with state $\psi(z)=\hat{\psi}_{m}(z)$, of the operator $O_{3}(0)=K(0)$ [Giachetti and Grecchi 2016a] transformed by the analytic translation $x \rightarrow x+i y$ :

$$
O_{3}(y, 0)=p^{2}+i(x+i y)^{3}=p^{2}+i\left(x^{2}-3 y^{2}\right) x+y^{3}-3 y x^{2}=p^{2}+V_{y}(x) .
$$

Then

$$
\begin{aligned}
& -\Im\left[\bar{\psi}(x+i y) \partial_{x} \psi(x+i y)\right]=\int_{x}^{\infty} \Im\left(V_{y}(s)-E\right)|\psi(s+i y)|^{2} d s \\
& =\int_{x}^{\infty}\left(s^{2}-3 y^{2}\right) s|\psi(s+i y)|^{2} d s=-\int_{-\infty}^{x}\left(s^{2}-3 y^{2}\right) s|\psi(s+i y)|^{2} d s \neq 0
\end{aligned}
$$

for $|x| \geq \sqrt{3}|y|, y \in \mathbb{R}$. In this case we have a rigorous confinement of the nodes in the region

$$
\mathbb{C}_{\sigma}=\{z=x+i y: y<0,|x|<-\sqrt{3} y\} \subset \mathbb{C}_{-}=\{z \in \mathbb{C}: \Im z<0\} .
$$

Now we extend the Loeffel-Martin method to the case $\Im E \neq 0, x=0$, obtaining the equation

$$
\Im\left(\hbar^{2} \bar{\phi}(y) \partial_{y} \phi(y)\right)=-\Im E \int_{y}^{\infty}|\phi(s)|^{2} d s \quad \text { for all } y \in \mathbb{R},
$$

where $\phi(y)=\psi(i y)$, if the integral in (1-2) exists and is bounded. Thus, the state of a nonreal level is free of imaginary zeros.

1c. The exact quantization condition [Giachetti and Grecchi 2016b]. We consider the semiclassical Hamiltonian

$$
H_{\hbar}=\hbar^{2} p^{2}+V(x), \quad V(x)=i\left(x^{3}-x\right), p^{2}=-\frac{d^{2}}{d x^{2}}, \hbar>0 .
$$

Let us fix $\hbar>0$ small, the level $E=E_{n}^{+}(\hbar) \in \mathbb{C}_{-}$(the case $E_{n}^{-}(\hbar)$ is perfectly analogous $)$ and the state $\psi_{E}(z)=\psi_{n}^{+}(\hbar, z)$ with $n$ nodes in $\mathbb{C}^{+}=\{z \in \mathbb{C}: \Re z>0\}$. We have the exact quantization condition

$$
J(E, \hbar):=\frac{\hbar}{2 i \pi} \oint_{\gamma} \frac{\psi_{E}^{\prime}(z)}{\psi_{E}(z)} d z+\frac{\hbar}{2}=\hbar\left(n+\frac{1}{2}\right),
$$


where $\gamma=\partial \Omega \subset \mathbb{C}^{+}$and $\Omega$ is a connected domain large enough to contain all the nodes. The fixed number $n$ and the isolation of the nodes allow us to bound the energy value.

In particular, for small $\hbar>0$, and fixed $n \in \mathbb{N}$, the quantization rules (1-4) become the semiclassical quantization conditions for $E=E_{n}^{+}(\hbar)$

$$
J(E, \hbar)=\frac{1}{2 i \pi} \oint_{\gamma} p_{0}(E, z) d z+O\left(\hbar^{2}\right)=\hbar\left(n+\frac{1}{2}\right),
$$

where $p_{0}(E, z)=\sqrt{V(z)-E}$, and the path $\gamma$ shrinks around the short Stokes line.

1d. The generalized changes of representation [Aguilar and Combes 1971]. A change of representation is given by a unitary transform $U_{\delta} \neq U_{0}=I$, where $\delta$ is any nonzero real number and $I$ is the identity operator, giving a new Hamiltonian $H_{\delta}=U_{\delta} H U_{\delta}^{*}$ unitarily equivalent to the original one. In this case, a level of the Hamiltonian is the same as that of the original one, $E_{n}(\delta)=E_{n}(0)$, and the state is transformed into $\psi_{n}(\delta)=U_{\delta} \psi_{n}$. Let $H_{\delta}$ be an analytic family of operators of type A in the parameter $\delta \in \Omega \subset \mathbb{C}$, with $\mathbb{R} \subset \Omega$, and let $H$ have discrete spectrum $\left\{E_{n}\right\}_{n}$. Then the operator $H_{\delta}, \delta \in \Omega \subset \mathbb{C}$, is isospectral to $H:=H_{0}$ so that

$$
E_{n}(\delta)=E_{n},
$$

and the vector $\psi_{n}(\delta)=U_{\delta} \psi_{n}, \delta \in \Omega$ and $\delta=i \theta, \theta \in \mathbb{R}$, is the state $\psi_{n}$ in a generalized representation.

Actually, there are more general changes of representation with definite transformations of the eigenvalues

$$
E_{n}(\delta)=f_{\delta}\left(E_{n}\right)
$$

1e. The Stokes sectors, the asymptotic behavior, the Riccati equation [1761] and the semiclassical series expansion. We recall the asymptotic behavior of the states at infinity, the Stokes sectors and the Carlini semiclassical series expansion [Plana 1832; Fröman and Fröman 1965].

Let us fix a direction in the complex plane of the variable $\arg (z)=\alpha$. The asymptotic behavior of a fundamental solution of $H_{\hbar}$ (see (1-3)) at infinity in this direction is given by [Sibuya 1975]

$$
\hbar \lim _{\substack{|z| \rightarrow \infty \\ \arg z=\alpha}} \frac{\ln \psi_{n}(z)}{\int^{z} \sqrt{V(w)} d w}= \pm 1 .
$$

The Stokes directions $\alpha_{j}$ are defined by the oscillatory condition of the fundamental solutions at infinity:

$$
\lim _{|z| \rightarrow \infty} \arg \left(\ln \psi_{n}(z)\right)=\pi / 2+j \pi, \quad j=-2,-1,0,1,2 .
$$


A Stokes sector of the complex plane is a minimal sector between two Stokes directions. In the case of the cubic oscillator $H_{\hbar}$, the Stokes directions $\arg (z)=\alpha_{j}$ are

$$
\lim _{|z| \rightarrow \infty} \arg \left(\ln \psi_{n}(z)\right)=5 \arg (i z) / 2=\pi / 2+j \pi, \quad j=-2,-1,0,1,2,
$$

and the Stokes sectors

$$
S_{j}=\{z \in \mathbb{C}:|\arg (i z)-2 j \pi / 5|<\pi / 5\}, \quad j=-2,-1,0,1,2 .
$$

A state $\psi_{n}(\hbar)$ of the Hamiltonian $H_{\hbar}$ is exponentially decreasing at infinity in the sectors $S_{ \pm 1}$ and exponentially increasing in the sectors $S_{0}$ and $S_{ \pm 2}$.

This state has large zeros only in the imaginary asymptotic direction. If we label the zeros $Z_{j}$ such that $\left|Z_{j}\right| \rightarrow \infty$ for $j \rightarrow \infty$,

$$
\arg \left(Z_{j}\right) \rightarrow \alpha_{ \pm 2}=\pi / 2 \quad \text { as } j \rightarrow \infty .
$$

Remark. In the case of a double-well cubic oscillator, this result is not sufficient for our purposes. We have proven that in the case of positive levels $E_{m}$ the large zeros of $\psi_{m}$ are exactly imaginary. Moreover, we have $\mp \Im E_{n}^{ \pm}>0$ and the large zeros of $\psi_{n}^{ \pm}$are in $\mathbb{C}^{\mp}$.

We express two fundamental solutions, in a given sector $S_{j}$, of the Schrödinger equation

$$
\left(\hbar^{2} p^{2}+p_{0}^{2}(z)\right) \psi(z)=0, \quad p^{2}=-\frac{d^{2}}{d z^{2}}, p_{0}^{2}(z)=V(z)-E,
$$

in the form

$$
\psi_{ \pm}(z)=\exp \left( \pm \frac{1}{\hbar} \int_{0}^{z} p_{\hbar}(w) d w\right)
$$

where $p_{\hbar}(z)$ satisfies the Riccati equation

$$
p_{\hbar}^{2}(z)+\hbar p_{\hbar}^{\prime}(z)=p_{0}^{2}(z), \quad p_{0}^{2}(z)=V(z)-E .
$$

We formally solve by the Carlini series

$$
p_{\hbar}(z) \sim \sum_{n} p_{n}(z) \hbar^{n},
$$

where the coefficients are computed recursively starting from the positive definition of the classical momentum: $p_{0}(z)=\sqrt{V(z)-E}$ and

$$
\begin{aligned}
& p_{1}(z)=\frac{i p_{0}^{\prime}(z)}{2 p_{0}(z)} \\
& p_{n}(z)=-\frac{1}{2 p_{0}(z)}\left(\sum_{j=1}^{n-1} p_{n-j}(z) p_{j}(z)+i p_{n-1}^{\prime}(z)\right), \quad n=2,3, \ldots
\end{aligned}
$$


Defining

$$
p_{\hbar}(z)=P_{\chi}(z)+\hbar Q_{\chi}(z), \quad \chi=\hbar^{2},
$$

we get the equation for $P_{\chi}(z)$

$$
P_{\chi}^{2}(z)-p_{0}^{2}(z)=-\chi\left(Q_{\chi}^{2}(z)+Q_{\chi}^{\prime}(z)\right)
$$

where

$$
Q_{\chi}(z)=-\frac{P_{\chi}^{\prime}(z)}{2 P_{\chi}(z)}
$$

We thus have the equivalent expression of the solutions

$$
\psi_{ \pm}(z)=\frac{1}{\sqrt{P_{\chi}(z)}} \exp \left( \pm \frac{1}{\hbar} \int_{0}^{z} P_{\chi}(w) d w\right),
$$

where the Riccati solution $P_{\chi}(z)$ has the even part of the Carlini expansion:

$$
P_{\chi}(z) \sim \sum_{j \in \mathbb{N}} \chi^{j} p_{2 j}(z) \quad \text { with truncations } P_{\chi}^{N}(z) \sim \sum_{j}^{N} \chi^{j} p_{2 j}(z) .
$$

Thus, the semiclassical momentum $P_{\chi}(z)$ is an asymptotic notion, and the coefficients of the Carlini series are singular near the turning points. In certain cases, the exact momentum $P_{\chi}(z)$ is the Borel sum of the series in (1-13) [Voros 1994; Delabaere et al. 1997]. Notice that the behaviors of the fundamental solutions, for large $|z|$ in a Stokes sector, are given by (1-12) at a zeroth order of approximation with $p_{0}(z)$ in place of $P_{\chi}(z)$ [Sibuya 1975].

1f. The Stokes lines and the classical trajectories. Here we also examine the critical energies at the semiclassical limit $\hbar=0$.

Let us consider the real cubic oscillator

$$
H_{r}(\hbar):=\hbar^{2} p^{2}+V^{r}(x), \quad V^{r}(x)=x^{3}-x, \hbar>0,
$$

and the energy range $R_{0}=\left[-E_{0}, E_{0}\right]$, where $E_{0}=2 / 3 \sqrt{3}$. The union of the trajectories of the classical motion $M(E)$ consists of the oscillation range $\sigma(E):=$ $\left[I_{-}, I_{+}\right]$and the escape route $B(E):=\left(-\infty, I_{0}\right], I_{0}<-1 / \sqrt{3}<I_{-}<I_{+}$. We recall the definition of the Stokes lines at energy $E$. A Stokes line is tangent at each one of its points $z$ to the direction $d z^{2}(z)$, where $p(z)^{2} d z^{2}(z)<0, p(z)^{2}=V^{r}(z)-E$, and starts from a turning point $I$. From the complex semiclassical point of view, $\sigma(E)$ is the internal Stokes line and $B(E)$ is the exceptional Stokes line [Giller 2011].

The fundamental state $\psi_{E}^{0}(\hbar, x), E \in R_{0}$, subdominant in the positive semiaxis,

$$
\psi_{E}^{0}(\hbar, x) \sim \frac{1}{\sqrt{p(x)}} \exp \left(-\frac{1}{\hbar} \int_{I_{+}}^{x} p(w) d w\right),
$$


for large $x>0$, can be continued onto the complex plane cut along $\sigma(E)$ and $B(E)$. We extend the approximate fundamental state by setting $\psi_{E}^{0}(\hbar, x)=\left(\psi_{E}^{0}(\hbar, x+i 0)+\right.$ $\left.\psi_{E}^{0}(\hbar, x-i 0)\right) / 2$ for all $x \in M(E)$. This approximation oscillates in $M(E)$ as the exact fundamental state. In the limit $\hbar \rightarrow 0$ all the zeros of the exact fundamental state $\psi_{E}(\hbar)$ tend to $M(E)$ as $\hbar \rightarrow 0$ [Giller 2011]. The union of the trajectories of the classical motion $M(E)$ is singular at $E=E_{0}=2 / 3 \sqrt{3}$, where the escape route $B(E)$ touches the oscillation range $\sigma(E)$.

Now we consider the $P T$-symmetric double well with Hamiltonian $H_{\hbar}=\hbar^{2} p^{2}+$ $i\left(x^{3}-x\right)$, for $\hbar>0$. For $E>0$ large, the escape route is part of the imaginary axis, while the oscillation range should be computed numerically.

The union of the trajectories of the classical motion $M(E)$ has the singular point $E^{c}=0.352268 \ldots$ where the escape route touches the oscillation range at its middle point. For larger $E>E^{c}$ the escape route and the oscillation range are separated. The energy levels $E_{n}(\hbar)$ are positive for large $\hbar>0$ and are imaginary at the limit $\hbar \rightarrow 0$. This means the existence of (at least) a singularity and a crossing at $\hbar_{n}>0$ for each pair of functions $E_{n}^{ \pm}(\hbar), 0<\hbar<\hbar_{n}$. The crossing parameters $\hbar_{n}>0$ and the energy crossings $E_{n}^{c}>0$ have the limits $\hbar_{n} \rightarrow 0$ and $E_{n}^{c}=E_{n}^{ \pm}\left(\hbar_{n}\right) \rightarrow E^{c}$ as $n \rightarrow \infty$. The approximate actions around the oscillation range defined at the crossing, $J_{n}:=n \hbar_{n}$, have limit $J\left(E^{c}, 0\right)$ as $n \rightarrow \infty$ (see (1-5)), the action integral around the oscillation range $\sigma(E)$.

We now consider the two cases denoted by the signs \pm together. At energies $E_{n}^{\mp}(0)= \pm i E_{0}$, the oscillation range $\sigma(E)$ reduces to the points $I_{-}=I_{+}=\mp 1 / \sqrt{3}$, respectively. It is possible to prove that the escape route $B(E)$ starts at $I_{0}= \pm 2 / \sqrt{3}$ and goes toward $+i \infty$ staying in the half-plane $\mathbb{C}^{ \pm}$. Moreover, the levels $E_{n}^{ \pm}(\hbar)$ are nonreal for $\hbar<\hbar_{n}$ and the zeros of $\psi_{n}^{ \pm}(\hbar)$ cannot be on the imaginary axis. Thus, the $n$ nodes of $\psi_{n}^{ \pm}(\hbar)$ are stable in $\mathbb{C}^{\mp}$ for $0<\hbar<\hbar_{n}$.

1g. The theory of the regular perturbations. Let $H_{0}$ be a self-adjoint operator on the Hilbert space $\mathscr{H}$ with compact resolvent, so that the spectral theorem takes the expression

$$
H_{0}=\sum_{n} E_{n} P_{n}, \quad \mathbf{1}=\sum_{n} P_{n}, P_{n} P_{k}=\delta_{n}^{k} P_{n}, P_{n}^{*}=P_{n},
$$

where the set of real eigenvalues $\left\{E_{n}\right\}_{n}$ (the spectrum of $\left.H_{0}: \sigma\left(H_{0}\right)\right)$ have no accumulation points in $\mathbb{R}$ and the orthogonal projectors $P_{n}$ have a finite-dimensional image: $m_{n}=\operatorname{dim}\left(P_{n} \mathscr{H}\right) \in \mathbb{N}$.

Resolvent. For $z \in \mathbb{C}-\sigma\left(H_{0}\right)$ the resolvent of $H_{0}$ with parameter $z$,

$$
R_{0}(z)=\left(H_{0}-z\right)^{-1}=\sum_{n}\left(E_{n}-z\right)^{-1} P_{n}
$$


is a compact operator, with norm

$$
\left\|R_{0}(z) u\right\|^{2} \leq \max _{n}\left|E_{n}-z\right|^{-2} \sum_{n}\left\|P_{n} u\right\|^{2}=\|u\|^{2} / \rho\left(z, \sigma\left(H_{0}\right)\right)^{2},
$$

where $\rho\left(z, \sigma\left(H_{0}\right)\right)$ is the distance of $z$ from the spectrum of $H_{0}$. In particular, if $u=P_{j} u$, we have

$$
\left\|R_{0}(z) u\right\|=1 / \rho\left(z, E_{j}\right) .
$$

Operator $V$ relatively bounded with respect to $H_{0}(\mathrm{Kato})$. Let $V$ be another operator on $\mathscr{H}$ such that $D\left(H_{0}\right) \subset D(V)$ and

$$
\|V u\| \leq a\left\|H_{0} u\right\|+b\|u\| \quad \text { for all } u \in D\left(H_{0}\right)
$$

for positive constants $a$ and $b$. We use the notation $V<H_{0}$.

Theorem. With $V$ as above, the operator

$$
A:=A(z):=V R_{0}(z)
$$

for $z \notin \sigma\left(H_{0}\right)$ is a bounded operator.

Proof. Let us consider any $v \in \mathscr{H}$; then $A v=V u$, where $u=R_{0}(z) v$, with norm $\|u\| \leq\left\|R_{0}(z)\right\|\|v\| \equiv c\|v\|$. For the relative boundedness we have $\|V u\| \leq$ $a\left\|H_{0} u\right\|+b\|u\|$, where $\left\|H_{0} u\right\|=\left\|H_{0} R_{0}(z) v\right\|=\|v+z u\| \leq\|v\|+|z|\|u\|$; hence, $\|A v\| \leq(a(1+|z| c)+b c)\|v\|$, whence $A$ is a bounded operator with norm

$$
\|A\| \leq a(1+|z| c)+b c .
$$

Analytic family of operators. Let us consider the analytic family of operators $H_{\epsilon}=$ $H_{0}+\epsilon V$ for $\epsilon \in \mathbb{C}$.

Theorem. The resolvent of $H_{\epsilon}, R_{\epsilon}(z)=B_{\epsilon}$ for a fixed $z \notin \sigma\left(H_{\epsilon}\right)$, is an analytic family of bounded operators on the domain $|\beta|<b_{0}$.

Proof. We have

$$
B_{\epsilon}=R_{\epsilon}(z)=\left(\left(H_{0}-z\right)+\epsilon V\right)^{-1}=R_{0}(z)(1+\epsilon A)^{-1}=R_{0}(z) \sum_{n}(-\epsilon A)^{n},
$$

where $A$ is defined in (1-15) and the series of powers of operators converges in norm for $|\epsilon|<b_{0}=1 /\|A\|$.

Norm convergence of the resolvents. We have that

$$
\left\|B_{\epsilon}-B_{0}\right\| \leq\left\|R_{0}(z)\right\| \sum_{n=1}^{\infty}(|\epsilon|\|A\|)^{n}
$$

vanishes as $\epsilon \rightarrow 0$. Analogously, for $b_{1}>0$ small and $\left|\epsilon_{1}\right|<b_{1},\left\|B_{\epsilon}-B_{\epsilon_{1}}\right\| \rightarrow 0$ as $\epsilon \rightarrow \epsilon_{1}$. 
Perturbation not relatively bounded.

Quadratic estimate 1. For any $u \in D\left(H_{0}\right) \cap D(V)$, there exist $a, b>0$ such that

$$
\left\|H_{0} u\right\|^{2}+|\epsilon|^{2}\|V u\|^{2} \leq a\left\|H_{\epsilon} u\right\|^{2}+b\|u\|^{2} .
$$

Let $V=V_{0} V^{\prime}$ such that $V^{\prime}<H$ and $V_{0}=V_{0}^{*}<H_{0}$.

Quadratic estimate 2. For any $u \in D\left(H_{0}\right) \cap D(V)$, there exist positive constants $a$ and $b$ such that

$$
\left\|V^{\prime} u\right\|^{2} \leq a\left\|H_{\epsilon} u\right\|^{2}+b\|u\|^{2} .
$$

We have

$$
R_{\epsilon}-R_{0}=\epsilon R_{0} V R_{\epsilon}=\epsilon A_{0} A^{\prime},
$$

where the $z \notin \sigma\left(H_{0}\right)$ dependence is omitted. Both the operators $A_{0}=R_{0} V_{0}$ and $A^{\prime}=V^{\prime} R_{\epsilon}$ are uniformly bounded as $\epsilon \rightarrow 0$. Thus, we have the norm resolvent convergence

$$
\left\|R_{\epsilon}-R_{0}\right\| \leq|\epsilon|\|A\|\left\|A^{\prime}\right\| \rightarrow 0
$$

as $\epsilon \rightarrow 0$.

In our case, we have $H_{0}=p^{2}+\alpha x^{2},|\alpha|=1, V_{0}=x, V^{\prime}=i x^{2}$ and $\epsilon=\sqrt{b} \geq 0$.

Projector on an isolated part of the spectrum. Let $\gamma=\partial \Gamma$ be a regular closed positively oriented curve in $\mathbb{C}$ encompassing a simple isolated eigenvalue of $H_{0}$ once:

$$
\sigma_{\Gamma}:=E_{j}=\Gamma \cap \sigma\left(H_{0}\right), \quad \gamma \cap \sigma\left(H_{0}\right)=\varnothing .
$$

For instance, let $\gamma$ be the circle $\left|z-E_{j}\right|=d / 2$, where $d>0$ is the isolation distance of $E_{j}$. We have

$$
P_{j}=-(1 / 2 \pi i) \int_{\gamma} R_{0}(z) d z
$$

because of (1-14) and the residue theorem. Because of the norm convergence of the resolvents and $\gamma$ being a compact set, we have the norm convergence of the projectors $P_{j}(\epsilon):=P_{\epsilon}$,

$$
\left\|P_{\epsilon}-P_{j}\right\| \rightarrow 0
$$

for $|\epsilon| \rightarrow 0$, and there exists $b_{0}>0$ such that

$$
P_{j} \epsilon=-(1 / 2 \pi i) \int_{\gamma} R_{\epsilon}(z) d z
$$

for $0<\epsilon<b_{0}$. 
Perturbation expansion of $P_{\epsilon}$. The set of projectors $P_{\epsilon}$ is an analytic family of projectors, and its power series expansion is

$$
P_{\epsilon}=\sum_{n} \epsilon^{n} Q_{n}
$$

where

$$
Q_{n}=-(2 \pi i)^{-1} \int_{\gamma} R_{0}(z)\left(-V R_{0}(z)\right)^{n} d z .
$$

Continuity and stability of the spectrum. Let $m_{\epsilon}=\operatorname{dim}\left(P_{\epsilon} \mathscr{H}\right) \in \mathbb{N}$ be the total multiplicity of the eigenvalues of $H_{\epsilon}$ contained in $\Gamma$. This is a continued function for $0<\epsilon \leq \epsilon_{0}$ and, being discrete-valued, is constant. This means that in $\Gamma$ we always have the only eigenvalue $E_{j}(\epsilon)$ near $E_{j}$, for $\epsilon$ small, and we can compute this analytic eigenvalue by the perturbation method.

1h. Perturbation expansion of a simple isolated eigenvalue. Let $E(0)$ be a simple isolated eigenvalue of $H_{0}$, with normalized eigenvector $u_{0}$ and projector $P_{0}$, so that $H_{0} u_{0}=E(0) u_{0}$ and $P_{0} u_{0}=u_{0}$. We have $\left\|P_{\epsilon}-P_{0}\right\| \rightarrow 0$ as $\epsilon \rightarrow 0$ so that $u_{\epsilon}=P_{\epsilon} u_{0} \rightarrow u_{0}$ as $\epsilon \rightarrow 0$ is an eigenvector: $H_{\epsilon} u_{\epsilon}=E(\epsilon) u_{\epsilon},\left\|u_{\epsilon}\right\|>0$. Thus, $\left\langle u_{0}, P_{\epsilon} u_{0}\right\rangle=\left\langle P_{\epsilon} u_{0}, P_{\epsilon} u_{0}\right\rangle \rightarrow 1$ as $\epsilon \rightarrow 0$. We can compute $E(\epsilon)$ by the expression

$$
\begin{aligned}
E(\epsilon) & =\left\langle u_{0}, H_{\epsilon} P_{\epsilon} u_{0}\right\rangle /\left\langle u_{0}, P_{\epsilon} u_{0}\right\rangle \\
& =E(0)+\epsilon\left\langle u_{0}, V P_{\epsilon} u_{0}\right\rangle /\left\langle u_{0}, P_{\epsilon} u_{0}\right\rangle \\
& =E(0)+\epsilon \frac{\sum_{n} \epsilon^{n}\left\langle u_{0}, V Q_{n} u_{0}\right\rangle}{\sum_{n} \epsilon^{n}\left\langle u_{0}, Q_{n} u_{0}\right\rangle} \\
& =E(0)+\epsilon\left\langle u_{0}, V u_{0}\right\rangle+O\left(\epsilon^{2}\right)=\sum_{n} \epsilon^{n} c_{n},
\end{aligned}
$$

where the $\left\{c_{n}\right\}_{n}$ are the coefficients of the power series expansion given by the perturbation theory as a ratio of two power series. Since the two series are geometric, it is possible to evaluate the remainder, and it turns out to be of the same order as the next perturbation coefficient.

1i. Divergent perturbation series. If $V$ is not bounded with respect to $H_{0}$, but any vector $V Q_{n} u_{0}$ or $Q_{n} u_{0}, n \in \mathbb{N}$, is bounded, we have bounded coefficients of a divergent perturbation series. We do not use the estimates of the growing of the coefficients and the remainders made with the perturbation theory because we have the exact behavior from the Stieltjes property and the behavior of the imaginary part of the "resonances".

Now it is useful to recall the method of summing the divergent perturbation series. 


\section{Divergent power series regular sum: a short story}

The formal series [Akhiezer 1965] as a vector function with specified terms

$$
\tilde{S}(a):=\sum_{k=0}^{\infty} a_{k}:=\sum a_{k}, \quad a=\left\{a_{k}\right\}_{k \in \mathbb{N}}, a_{k} \in \mathbb{C},
$$

is convergent (Cauchy) with sum $S(a)$, if there exists

$$
S(a)=\lim _{n \rightarrow \infty} S_{n}(a) \in \mathbb{C}, \quad S_{n}(a)=\sum_{k=0}^{n} a_{k} .
$$

Actually Pietro Mengoli's [1650] idea of a convergent series was not so different.

For the simple formal series $\tilde{S}(a)$ as a function of the sequence of its terms

$$
a=\left\{a_{k}=(-1)^{k}\right\}_{k \in \mathbb{N}},
$$

Guido Grandi [1703], obviously unaware of the Cauchy condition, suggested that the only possible sum of the series $S(a)$ is $\frac{1}{2}$.

Let us recall a possible proof.

We assume that the sum $S(a)$ is a linear functional of the vector $a$. Now we recall some obvious facts.

The sum of a series with only one nonzero term must exist:

$$
\text { if } b=b_{0}, 0,0,0, \ldots, \quad S(b)=b_{0} .
$$

The invariance of the series by translation of the indexes is obvious:

$$
\tilde{S}(T a)=\tilde{S}(a), \quad a=a_{0}, a_{1}, \ldots, T a=0, a_{0}, a_{1}, \ldots
$$

So we must admit the same invariance for the sum

$$
S(T a)=S(a) .
$$

The proof that $S(a)$, for $a$ as in (2-2), if it exists, must be equal to $\frac{1}{2}$, is based on the extension of the linearity of the sum used by Pietro Mengoli for convergent series.

Hypothesis 1. The sum of a series $S(a)$ is a linear function of the vector of its elements $a$ :

$$
S(a)=S(b)+\lambda S(c), \quad a=b+\lambda c,
$$

if all the sums exist.

Proposition. Assuming the existence of the sum S(a) of the formal series (2-2),

$$
S(a)=\frac{1}{2} .
$$


Proof. We decompose the vector $a$ as $a=b+a_{0}^{\prime}$, where $b=a_{0}, 0,0, \ldots$ and $a_{0}^{\prime}=0, a_{1}, a_{2}, \ldots=-T a$. Thus, since $a=b+\lambda T a$ where $\lambda=-1$, by Hypothesis 1 ,

$$
S(c)=S(b)+\lambda S(T a)=b_{0}+\lambda S(a),
$$

if $\lambda \in \mathbb{C}, c=b+\lambda T a$ and $b$ is as above in (2-3). The sum of the series satisfies a linear equation

$$
S(a)=S(b)-S(T a)=a_{0}-S(a)=1-S(a),
$$

with the unique solution $S(a)=\frac{1}{2}$.

Notice that Mangione [1971] says that the statement of the existence of the sum of the series $\tilde{S}(a)$ is a mistake (obviously it is, assuming the Cauchy definition of the sum).

Later Cesaro proposed his method of the sum (1894-1897). The partial sums of $\tilde{S}(a)$ are

$$
S_{n}(a)=\frac{1}{2}+\frac{1}{2}(-1)^{n}, \quad n=0,1,2, \ldots,
$$

so that the mean values of the first sums are

$$
S_{N}^{c}(a)=\sum_{n=0}^{N-1} \frac{S_{n}(a)}{N}=\frac{1}{2}+\frac{1}{2 N}(-1)^{N-1}, \quad N=1,2, \ldots
$$

The Cesaro sum of the series, $\lim _{N \rightarrow \infty} S_{N}^{c}(a)$, exists and coincides with Grandi's.

2a. The Taylor expansion of a function. An analytic function is the sum of its power series expansion on a disk:

$$
f(z)=\sum_{k=0}^{\infty} c_{k} z^{k}, \quad c_{k}=\frac{f^{(k)}(0)}{k !},\left|c_{k}\right|=\frac{1}{r_{k}^{k}},|z|<r,
$$

where $r=\underline{\lim } r_{k}>0$.

Example 1. We have the expansion

$$
f(z)=\frac{1}{1+z}=\sum_{k=0}^{\infty} c_{k} z^{k}=\sum_{k=0}^{\infty} c_{k} z^{-k-1}, \quad c_{k}=(-1)^{k},
$$

on the open disk $|z|<1$. Extending the sum by continuity to the point $z=1$, we get the Grandi proof of the Grandi sum of the Grandi series.

We now state some definitions and results. 
2b. A divergent power series asymptotic to a function (Poincaré). A divergent power series is asymptotic to a function $f$ when

$$
\left|f(x)-\sum_{n=0}^{N-1} c_{n} x^{n}\right| \leq C_{N} x^{N}, \quad 0<x<X .
$$

The astronomer approximants. Let $C_{N}=a^{N} N$ !, where $a>0$. For any $x$ as above, we fix $N(x)=[1 / a x]$ and we take the approximant

$$
\sum_{n=0}^{N(x)-1} c_{n} x^{n}
$$

with an error exponentially small as $x \rightarrow 0$.

Remark. The asymptotic function $f(x)$ in (2-10) is not unique; there is also the family of functions $f_{\epsilon}(x)=f(x)+\epsilon \exp (-1 / a x)$ for $\epsilon \neq 0$ with $|\epsilon|$ small enough.

2c. Strong asymptotics and uniqueness of the function (Carleman). Consider the formal power series $\sum_{n}^{\infty} c_{n} z^{n}$ and the function $f(z)$. If there exist $X, C_{N}>0$ such that

$$
\sum_{N} \frac{1}{\sqrt[N]{C_{N}}}=\infty
$$

and $f(z)$ is analytic on

with

$$
D_{X}=\{z:|z| \leq X,|\arg (z)| \leq \pi / 2\}
$$

$$
\left|f(z)-\sum_{n}^{N-1} c_{n} z^{n}\right| \leq C_{N}|z|^{N}
$$

uniformly for $N \in \mathbb{N}$ and $z \in D_{X}$, then the function $f(z)$ is uniquely defined on $D_{X}$ by the formal series and is called the sum of the series.

2d. Direct methods of sum. We define more general approximants of the series (2-8), not only the polynomials $S_{n}(z)=\sum_{k=0}^{n} c_{k} z^{k}$ but also the rational functions called diagonal Padé approximants:

$$
\frac{Q_{j}(z)}{P_{j}(z)}=S_{2 j}(z)+O\left(z^{2 j+1}\right)
$$

where $P_{j}(z)$ and $Q_{j}(z)$ are polynomials of degree $j$, with $P_{j}(0)=1$. If they converge, we say that the series is summable, and the limit is called the Padé sum. Let us notice that in the case (2-9) the approximants are exact:

$$
\frac{Q_{j}(z)}{P_{j}(z)}=f(z), \quad z \neq-1,
$$

for all $j \geq 1$.

Another regular method of sum is the Borel one. 
For the series of Example 1, we call the Borel transform the entire function defined by the convergent series

$$
f^{B}(z)=\sum_{k=0}^{\infty} \frac{c_{k}}{k !} z^{k}=\sum_{k=0}^{\infty} \frac{1}{k !}(-z)^{k}=\exp (-z),
$$

and we get the sum of the original series by the integral

$$
f(z)=\int_{0}^{\infty} f^{B}(t z) \exp (-t) d t=\int_{0}^{\infty} \exp (-t(1+z)) d t
$$

on the half-plane $\mathfrak{R z}>-1$.

Example 2 (Euler function (1770)). We may take

$$
f(z)=\int_{0}^{\infty} \frac{1}{1+z t} \exp (-t) d t=\sum_{k=0}^{\infty} c_{k} z^{k}, \quad c_{k}=(-1)^{k} k ! .
$$

In this case, the power series converges nowhere, but we understand by inspection that it is the Borel sum of the Borel transform, $f^{B}(z)=(1+z)^{-1}$, on the half-plane $\Re z>0$.

Remark. In this case, the Borel transform is not entire, and the analytic continuation of the Borel transform on the half-axis is not automatic. In any case, the Borel summability does not directly give convergent approximants, but it is not difficult to find them by mapping the region of analyticity of the Borel transform on a disk containing the origin.

Criterion of Borel summability of a series to a function (Nevanlinna) [Caliceti et al. 1986]. Let us consider the formal power series $\sum_{n}^{\infty} c_{n} z^{n}$ and the function $f(z)$. If there exist $X, C_{N}>0$ such that

$$
C_{N}=O\left(A^{N} N !\right)
$$

and the function is analytic and

$$
\left|f(z)-\sum_{n}^{N-1} c_{n} z^{n}\right| \leq C_{N}|z|^{N}, \quad D_{X}=\{z:|z-X|<X\}
$$

uniformly for $N$ and $z$, then the function is uniquely defined as the Borel sum of the formal series.

Returning to the problem, is it possible for the Padé approximants to do better? Yes! Stieltjes (1894) proved the convergence of the Padé approximants on all of the cut plane $\mathbb{C}_{c}=\{z \in \mathbb{C}: z \neq 0,|\arg (z)|<\pi\}$. Our function can be written $f(z)=y g(y)=y \int_{0}^{\infty} \frac{1}{y+t} \exp (-t) d t=\int_{0}^{\infty} \frac{1}{1+t z} \exp (-t) d t=\sum_{j}(-1)^{j} c_{j} z^{j}$, 
where $y=1 / z$ and $g(y)$ is a Stieltjes function with the associated continued fraction (Laguerre) [Wall 1948]

$$
g(y)=1 /(y+1 /(1+1 /(y+2 /(1+2 /(y+3 /(\cdots)))))) .
$$

The approximants of the continued fraction are the Padé approximants of the series.

Definition. A Stieltjes function is a bounded analytic function on the cut plane, real for $z>0$, with $\overline{g(z)}=g(\bar{z})$, which satisfies the Herglotz property [Shohat and Tamarkin 1943, Lemma 2.2],

$$
\frac{\Im g(z)}{\Im z}<0, \quad \Im z \neq 0,
$$

vanishing at $\infty$. In this case, by the Stieltjes-Stone inversion formula, the nondecreasing measure $\mu(t)$ with $\mu(-\infty)=\mu(0)=0$ and $\mu(t)=(\mu(t-0)+\mu(t+0)) / 2$ is defined by

$$
\mu(b)-\mu(a)=\lim _{\epsilon \rightarrow 0}-\frac{1}{2 i \pi} \int_{a}^{b}(g(t+i \epsilon)-g(t-i \epsilon)) d t,
$$

for any $a$ and $b$ with $0 \leq a<b$, such that

$$
g(z)=\int_{0}^{\infty} \frac{1}{z+t} d \mu(t):=S\left(\frac{1}{z+t}\right) .
$$

Definition of the series of Hamburger (more general than the Stieltjes one) and the Hamburger moment problem. Let us consider the real sequence $c:=\left\{c_{n}\right\}_{n \in \mathbb{N}}$. There exists a nondecreasing function $\mu$, taking on infinitely many values in the interval $[-\infty, \infty)$, such that the sequence $c$ is the sequence of the moments of the measure $d \mu$ :

$$
c_{n}=\int_{-\infty}^{\infty} t^{n} d \mu(t):=S\left(t^{n}\right) \in \mathbb{R} \quad \text { for all } n \in \mathbb{N} .
$$

The existence of a measure is proved if and only if we have the positivity of the sequence of $n \times n$ matrices

$$
\left(A_{n}\right)_{j, k}=c_{j+k}
$$

so that the determinants

$$
D_{n}=\operatorname{det} A_{n}>0 \quad \text { for all } n \in \mathbb{N} \text {. }
$$

We can set $c_{0}=D_{0}=D_{-1}=1$. 
2e. The Stieltjes series and the Stieltjes moment problem. Let us consider the real sequence $c:=\left\{c_{n}\right\}_{n \in \mathbb{N}}$. There exists a nondecreasing function $\mu$, taking on infinitely many values in the interval $[0, \infty)$, such that the sequence $c$ is the sequence of the moments of the measure $d \mu$ :

$$
c_{n}=\int_{0}^{\infty} t^{n} d \mu(t):=S\left(t^{n}\right) \in \mathbb{R} \quad \text { for all } n \in \mathbb{N} .
$$

The existence is proved if and only if we have the positivity of the two sequences of $n \times n$ matrices

$$
\left(A_{n}\right)_{j, k}=c_{j+k}, \quad\left(B_{n}\right)_{j, k}=c_{j+k+1}
$$

so that we have

$$
D_{n}=\operatorname{det} A_{n}>0 \quad \text { for all } n \in \mathbb{N} .
$$

We can set $c_{0}=D_{0}=D_{-1}=1$.

2f. Definition of orthonormal polynomials. The orthonormal polynomials $P_{n}(t)$ for the measure $d \mu$ and the mean $S$,

$$
S\left(P_{n} P_{m}\right)=\delta_{m}^{n},
$$

have an explicit expression:

$$
P_{n}(t)=\frac{1}{\sqrt{D_{n-1} D_{n}}} \operatorname{det}\left(\begin{array}{cccc}
c_{0} & c_{1} & \cdots & c_{n} \\
c_{1} & c_{2} & \cdots & c_{n+1} \\
\vdots & \vdots & \ddots & \vdots \\
c_{n-1} & c_{n} & \cdots & c_{2 n-1} \\
1 & t & \cdots & t^{n}
\end{array}\right)=\frac{\sqrt{D_{n-1}}}{\sqrt{D_{n}}} t^{n}+R_{n-1}(t) .
$$

It is easy to prove that

$$
S\left(P_{n}(t) t^{m}\right)=\sqrt{\left(D_{n} / D_{n-1}\right)} \delta_{n}^{m}
$$

if $m \leq n$. Let us expand

$$
t P_{n}(t)=a_{n, n+1} P_{n+1}(t)+a_{n, n} P_{n}(t)+a_{n, n-1} P_{n-1}(t)+\cdots .
$$

Comparing the leading coefficients, we get

$$
a_{n, n+1}=\frac{\sqrt{D_{n-1} D_{n+1}}}{D_{n}} .
$$

Multiplying by $P_{m}(t)$ and taking the mean, we get

$$
a_{n, n-j}=S\left(t P_{n}(t) P_{n-j}(t)\right)=0 \quad \text { for all } j>1, \quad a_{n, n-1}=a_{n-1, n} .
$$


Setting $b_{n}=a_{n, n+1}$ and $a_{n}=a_{n, n}$, we get the difference equation

$$
t P_{n}(t)=b_{n} P_{n+1}(t)+a_{n} P_{n}(t)+b_{n-1} P_{n-1}(t), \quad n \in \mathbb{N},
$$

with the boundary conditions given by $b_{-1}=0$ and $P_{0}(t) \equiv 1$.

2g. The determination of the moment problem and the essential self-adjointness of an operator! The determination of the Stieltjes moment problem can be seen as the essential self-adjointness of a positive operator $B$ in $\mathscr{H}$. Let us consider a generating vector $e_{0}$ on the domain of all the operators $D=\bigcap_{n} B^{n}$ and the linear space $L=L\left(B, e_{0}\right)$ generated by the vectors $\left\{e_{n}^{\prime}:=B^{n} e_{0}\right\}_{n}$ and the Hilbert subspace $\mathscr{H}\left(B, e_{0}\right)$, the closure of $L\left(B, e_{0}\right)$ in $\mathscr{H} . \dot{A}$ is $B$ restricted to $L$. If $\dot{A}$ is essentially self-adjoint, we call $A$ its positive self-adjoint closure in $\mathscr{H}\left(A, e_{0}\right)$ with spectral function $E(x), x \geq 0$. In this case, the unique measure $\mu(x)$ is the mean value of the spectral function on the generating vector $e_{0},\left\langle e_{0}, E(x) e_{0}\right\rangle$, and the Stieltjes function given by the mean value of the resolvent for $-z \in \mathbb{C}_{c}$,

$$
\begin{aligned}
g(z) & =-\left\langle e_{0}, \frac{1}{A-z} e_{0}\right\rangle=\int_{0}^{\infty} \frac{1}{z-x} d \mu(x) \\
& =\frac{c_{0}}{z}+\frac{c_{1}}{z^{2}}+\cdots+\frac{c_{2 n-1}}{z^{2 n}}+O\left(1 / z^{2 n+1}\right),
\end{aligned}
$$

for $|z|$ large, is the Stieltjes sum of its asymptotic series. Let us consider the infinite real Jacobian matrix as the representation of the linear operator $\dot{A}$ on the canonical basis $\left\{e_{n}=P_{n}(\dot{A}) e_{0}\right\}_{n}$ so that

$$
\dot{A} e_{k}=b_{k-1} e_{k-1}+a_{k} e_{k}+b_{k} e_{k+1}, \quad b_{k}>0, k \in \mathbb{N}, b_{-1}=0 .
$$

The operator $\dot{A}$ is defined as a symmetric operator on the dense set of finite vectors, $g_{N}=\sum^{N} x_{k} e_{k}$. We define the adjoint operator $\dot{A}^{*}$ by the condition $g=\sum x_{k} e_{k} \in$ $D\left(\dot{A}^{*}\right)$ if there exists $g^{*}=\sum y_{k} e_{k}$ such that

$$
\left\langle\dot{A} e_{k}, g\right\rangle=\left\langle e_{k}, g^{*}\right\rangle \quad \text { for all } k \in \mathbb{N} \text { with } g^{*}:=\dot{A}^{*} g
$$

or

$$
b_{k-1} x_{k-1}+a_{k} x_{k}+b_{k} x_{k+1}=y_{k} \quad \text { for all } k \in \mathbb{N} .
$$

Thus, the vector $g \in D\left(\dot{A}^{*}\right)$ if $g^{*}$ is in the space $l^{2}$ :

$$
\sum_{k}\left|b_{k-1} x_{k-1}+a_{k} x_{k}+b_{k} x_{k+1}\right|^{2}<\infty .
$$

The deficiency index of the operator $\dot{A}$ is $(0,0)$ or $(1,1)$.

We look for complex eigenvalues of $\dot{A}^{*}$ :

$$
\begin{aligned}
\dot{A}^{*} g & =\sum\left(b_{k-1} x_{k-1}+a_{k} x_{k}+b_{k} x_{k+1}\right) e_{k} \\
& =\sum x_{k}\left(b_{k-1} e_{k-1}+a_{k} e_{k}+b_{k} e_{k+1}\right)=\lambda \sum x_{k} e_{k}:=\lambda g
\end{aligned}
$$


so that

$$
\lambda x_{k}=b_{k-1} x_{k-1}+a_{k} x_{k}+b_{k} x_{k+1}, \quad k \in \mathbb{Z}_{+},
$$

with boundary conditions $\lambda x_{0}=a_{0} x_{0}+b_{0} x_{1}$ or $x_{1}=\left(\lambda-a_{0}\right) x_{0} / b_{0}, x_{0}=c \neq 0$, so that $x_{k}=c P_{k}(\lambda)$. The same recurrence relation (2-12), with initial conditions $Q_{0}(\lambda)=0$ and $Q_{1}(\lambda)=1 / b_{0}$, defines the polynomials $Q_{k}(\lambda)$.

It is equivalent to consider the relation

$$
z Y_{k+1}(z)=\left(z-a_{k}\right) Y_{k}(z)-b_{k-1}^{2} Y_{k-1}(z), \quad k \in \mathbb{Z}_{+},
$$

with solutions $N_{k}$ and $M_{k}$ for boundary conditions $N_{0}=Q_{0}=0, N_{1}=1, M_{0}=$ $P_{0}=1$ and $M_{1}=z-a_{0}$ :

$$
\begin{aligned}
M_{k}(z) & =b_{0} b_{1} \cdots b_{k-1} P_{k}(z), \quad N_{k}(z)=b_{0} b_{1} \cdots b_{k-1} Q_{k}(z), \quad k>0, \\
g(z) & =1 /\left(z-a_{0}-b_{0}^{2} /\left(z-a_{1}-b_{1}^{2} /\left(z-a_{2}-b_{2}^{2} /\left(z-a_{3}-\cdots\right)\right)\right)\right) \\
& =\frac{Q_{k}(z)}{P_{k}(z)}+O\left(1 / z^{2 k+1}\right) .
\end{aligned}
$$

In the determined case the sequence of diagonal Padé approximants converge for $z \in \mathbb{C}_{c}$ to the function

$$
g(z)=\lim _{k \rightarrow \infty}\left(\frac{N_{k}(z)}{M_{k}(z)} \equiv \frac{Q_{k}(z)}{P_{k}(z)}\right) .
$$

Thus, we have deficiency index $(0,0)$ if and only if

$$
\sum_{k}\left|P_{k}(\lambda)\right|^{2}=\infty
$$

We have the analogue of the Liouville-Ostrogradskii formula for $k \in \mathbb{Z}_{+}$:

$b_{k-1} P_{k-1} Q_{k}-P_{k} b_{k-1} Q_{k-1}=-b_{k} P_{k+1} Q_{k}+P_{k} b_{k} Q_{k+1}=b_{0} P_{0} Q_{1}-P_{1} b_{0} Q_{0}=1$

or

$$
P_{k-1} Q_{k}-P_{k} Q_{k-1}=P_{k-1} Q_{k-1}^{\prime}-P_{k-1}^{\prime} Q_{k-1}=\frac{1}{b_{k-1}},
$$

where

$$
Q_{k-1}^{\prime}=Q_{k}-Q_{k-1}, \quad P_{k-1}^{\prime}=P_{k}-P_{k-1} .
$$

Theorem (Hellinger). Let us fix $z, n \in \mathbb{Z}_{+}$and

$$
w_{n}(t):=w_{n}(z, t)=-\frac{Q_{n}(z)-t Q_{n-1}(z)}{P_{n}(z)-t P_{n-1}(z)}, \quad \Im(z) \neq 0,
$$

where $w_{n}(z, \infty)=w_{n-1}(z, 0)$. The images of $w_{n}: \mathbb{R} \rightarrow \mathbb{C}$ are nested circles of $n$-decreasing radii

$$
r=r(n, z)=\frac{1}{|\Im z|} \frac{1}{\sum_{k=0}^{n-1}\left|P_{k}(z)\right|^{2}}
$$


In the case of zero deficiency index, we have the limit point case: $r(n, z) \rightarrow 0$, as $n \rightarrow \infty$, and $w_{n}(z, t)$ have a $t$-independent limit as $n \rightarrow \infty$.

Theorem (criterion). Let

$$
\sum_{n} \frac{1}{b_{n}}=\infty
$$

The J-matrix is determinate: we have a unique solution of the moment problem and convergence of the continued fraction.

Proof. Use the formula (2-15):

$$
\begin{aligned}
\sum_{k=1}^{n} \frac{1}{b_{k-1}} & =\left|\sum_{k=1}^{n}\left(P_{k-1} Q_{k}-P_{k} Q_{k-1}\right)(\lambda)\right| \\
& \leq 2 \sqrt{\sum_{k=0}^{n}\left|Q_{k}^{2}(\lambda)\right| \sum_{k=0}^{n}\left|P_{k}^{2}(\lambda)\right|}
\end{aligned}
$$

Theorem (Carleman criterion). If

$$
\sum_{n} \frac{1}{\sqrt[2 n]{c_{2 n}}}=\infty
$$

then the Hamburger moment problem

$$
c_{k}=\int_{-\infty}^{\infty} x^{k} d \mu(x)
$$

is determinate.

Proof. From (2-12) we have

$$
b_{0} b_{1} \cdots b_{n-1} P_{n}(\lambda)=\lambda^{n}+O\left(\lambda^{n-1}\right)
$$

for $\lambda$ large. Hence,

$$
b_{0} \cdots b_{n-1} S\left(P_{n}^{2}\right)=\int_{-\infty}^{\infty} x^{n} P_{n}(x) d \mu(x) \leq \sqrt{c_{2 n}} \sqrt{S\left(P_{n}^{2}\right)},
$$

or since $S\left(P_{n}^{2}\right)=1$,

$$
b_{0} \cdots b_{n-1} \leq \sqrt{c_{2 n}}
$$

and

$$
\infty=\sum_{n} \frac{1}{\sqrt[2 n]{c_{2 n}}} \leq \sum_{n} \frac{1}{\sqrt[n]{b_{0} \cdots b_{n-1}}}<e \sum_{n} \frac{1}{b_{n}}
$$

for a general inequality of Carleman. 
The determined Hamburger moment problem corresponds to a determined Stieltjes one:

$$
c_{n}^{\prime}=\int_{0}^{\infty} y^{n} d \mu^{\prime}(y)
$$

where $c_{n}^{\prime}=c_{2 n}, y=x^{2}$ and

$$
d \mu^{\prime}(y):=d \mu(\sqrt{y})+d \mu(-\sqrt{y})
$$

is the unique solution.

\section{References}

[Aguilar and Combes 1971] J. Aguilar and J. M. Combes, "A class of analytic perturbations for one-body Schrödinger Hamiltonians”, Comm. Math. Phys. 22:4 (1971), 269-279.

[Akhiezer 1965] N. I. Akhiezer, The classical moment problem and some related questions in analysis, Oliver \& Boyd, Edinburgh, 1965.

[Caliceti et al. 1986] E. Caliceti, V. Grecchi, and M. Maioli, "The distributional Borel summability and the large coupling $\Phi^{4}$ lattice fields", Comm. Math. Phys. 104:1 (1986), 163-174.

[Delabaere et al. 1997] E. Delabaere, H. Dillinger, and F. Pham, "Exact semiclassical expansions for one-dimensional quantum oscillators", J. Math. Phys. 38:12 (1997), 6126-6184.

[Fröman and Fröman 1965] N. Fröman and P. O. Fröman, JWKB approximation: contributions to the theory, North-Holland, Amsterdam, 1965.

[Giachetti and Grecchi 2016a] R. Giachetti and V. Grecchi, "Bender-Wu singularities", J. Math. Phys. 57:12 (2016), 122109.

[Giachetti and Grecchi 2016b] R. Giachetti and V. Grecchi, "Level crossings in a $P T$-symmetric double well”, J. Phys. A 49:10 (2016), 105202.

[Giller 2011] S. Giller, "The semiclassical small- $\hbar$ limit of loci of roots of subdominant solutions for polynomial potentials", J. Math. Phys. 52:6 (2011), 063514.

[Grandi 1703] G. Grandi, Quadratura circuli, et hyperbola per infinitas hyperbolas, et parabolas geometricè exhibita, Francisci Bindi, Pisa, 1703.

[Kato 1966] T. Kato, Perturbation theory for linear operators, Grundlehren math. Wissenschaften 132, Springer, Berlin, 1966.

[Loeffel et al. 1969] J. J. Loeffel, A. Martin, B. Simon, and A. S. Wightman, "Pade approximants and the anharmonic oscillator", Phys. Lett. B 30:9 (1969), 656-658.

[Mangione 1971] C. Mangione, "Logica e fondamenti della matematica", Chapter 6, in Storia del pensiero filosofico e scientifico, vol. III: Il settecento, edited by L. Geymonat, Garzanti, Milan, 1971.

[Mengoli 1650] P. Mengoli, Nova quadratura arithmetica, Iacobi Monti, Bologna, 1650.

[Plana 1832] J. Plana, Théorie du mouvement de la lune, Imprimerie Royale, Turin, 1832.

[Reed and Simon 1975] M. Reed and B. Simon, Methods of modern mathematical physics, II: Fourier analysis, self-adjointness, Academic Press, New York, 1975.

[Reed and Simon 1978] M. Reed and B. Simon, Methods of modern mathematical physics, IV: Analysis of operators, Academic Press, New York, 1978.

[Riccati 1761] J. Riccati, Opere, Jacopo Giusti, Lucca, Italy, 1761. 
[Shohat and Tamarkin 1943] J. A. Shohat and J. D. Tamarkin, The problem of moments, Math. Surveys 1, American Mathematical Society, Providence, RI, 1943.

[Sibuya 1975] Y. Sibuya, "Distribution of zeros", Chapter 7, pp. 152-165 in Global theory of a second order linear ordinary differential equation with a polynomial coefficient, Math. Studies 18, North-Holland, Amsterdam, 1975.

[Voros 1994] A. Voros, "Exact quantization condition for anharmonic oscillators (in one dimension)", J. Phys. A 27:13 (1994), 4653-4661.

[Wall 1948] H. S. Wall, Analytic theory of continued fractions, Van Nostrand, New York, 1948.

Received 29 Sep 2016. Accepted 31 Oct 2016.

VINCENZO GRECCHI: vincenzo.grecchi@unibo.it

Dipartimento di Matematica, Università di Bologna, I-40126 Bologna, Italy

and

Gruppo Nazionale di Fisica Matematica, Istituto Nazionale di Alta Matematica Francesco Severi, I-00185 Roma, Italy 
EDITORIAL BOARD

ANTONIO CARCATERRA

ERIC A. CARLEN

FRANCESCO DELL'ISOLA

RAFFAELE ESPOSITO

ALBERT FANNJIANG

Gilles A. FranCFORT

Pierangelo MARCATI

JEAN-JACQUES MARIGO

PETER A. MARKOWICH

MARTIN OSTOJA-STARZEWSKI

PIERRE SEPPECHER

DAVID J. STEIGMANN

PAUl STEINMANN

PierRe M. SuQueT

MANAGING EDITORS

MICOL AMAR

CORRADO LATTANZIO

ANGELA MADEO

MARTIN OSTOJA-STARZEWSKI

ADVISORY BOARD

ADNAN AKAY

Holm AltenBaCH

MICOL AMAR

HARM ASKES

TEODOR ATANACKOVIĆ

VICTOR BERDICHEVSKY

GUY BOUCHITTÉ

ANDREA BRAIDES

ROBERTO CAMASSA

MAURO CARFORE

ERIC DARVE

FELIX DARVE

ANNA DE MASI

GianPiEtro DEL Piero

EMMANUELE Di BENEDETTO

BERNOLD FIEDLER

IRENE M. GAMBA

DAVID Y. GAO

SERGEY GAVRILYUK

TIMOTHY J. HEALEY

DOMINIQUE JEULIN

ROGER E. KHAYAT

CORRADO LATTANZIO

ROBERT P. LIPTON

ANGELO LUONGO

ANGELA MADEO

JUAN J. MANFREDI

CARLO MARCHIORO

GÉRARD A. MAUGIN

ROBERTO NATALINI PATRIZIO NEFF

ANDREY PIATNITSKI

ERRICO PRESUTTI

MARIO PULVIRENTI

LUCIO RUSSO

Miguel A. F. SANJUAN

PATRICK SElVADURAI

ALEXANDER P. SEYRANIAN

MIROSLAV ŠILHAVÝ

GUIDO SWEERS

ANTOINETTE TORDESILLAS

LEV TRUSKINOVSKY

JUAN J. L. VELÁZQUEZ VINCENZO VESPRI ANGELO VULPIANI msp.org/memocs

Università di Roma "La Sapienza", Italia

Rutgers University, USA

(CO-CHAIR) Università di Roma "La Sapienza", Italia

(TREASURER) Università dell'Aquila, Italia

University of California at Davis, USA

(CO-CHAIR) Université Paris-Nord, France

Università dell'Aquila, Italy

École Polytechnique, France

DAMTP Cambridge, UK, and University of Vienna, Austria

(CHAIR MANAGING EDITOR) Univ. of Illinois at Urbana-Champaign, USA

Université du Sud Toulon-Var, France

University of California at Berkeley, USA

Universität Erlangen-Nürnberg, Germany

LMA CNRS Marseille, France

Università di Roma "La Sapienza", Italia

Università dell'Aquila, Italy

Université de Lyon-INSA (Institut National des Sciences Appliquées), France

(CHAIR MANAGING EDITOR) Univ. of Illinois at Urbana-Champaign, USA

Carnegie Mellon University, USA, and Bilkent University, Turkey

Otto-von-Guericke-Universität Magdeburg, Germany

Università di Roma "La Sapienza", Italia

University of Sheffield, UK

University of Novi Sad, Serbia

Wayne State University, USA

Université du Sud Toulon-Var, France

Università di Roma Tor Vergata, Italia

University of North Carolina at Chapel Hill, USA

Università di Pavia, Italia

Stanford University, USA

Institut Polytechnique de Grenoble, France

Università dell'Aquila, Italia

Università di Ferrara and International Research Center MEMOCS, Italia

Vanderbilt University, USA

Freie Universität Berlin, Germany

University of Texas at Austin, USA

Federation University and Australian National University, Australia

Université Aix-Marseille, France

Cornell University, USA

École des Mines, France

University of Western Ontario, Canada

Università dell' Aquila, Italy

Louisiana State University, USA

Università dell'Aquila, Italia

Université de Lyon-INSA (Institut National des Sciences Appliquées), France University of Pittsburgh, USA

Università di Roma "La Sapienza”, Italia

Université Paris VI, France

Istituto per le Applicazioni del Calcolo "M. Picone", Italy

Universität Duisburg-Essen, Germany

Narvik University College, Norway, Russia

Università di Roma Tor Vergata, Italy

Università di Roma "La Sapienza”, Italia

Università di Roma “Tor Vergata”, Italia

Universidad Rey Juan Carlos, Madrid, Spain

McGill University, Canada

Moscow State Lomonosov University, Russia

Academy of Sciences of the Czech Republic

Universität zu Köln, Germany

University of Melbourne, Australia

École Polytechnique, France

Bonn University, Germany

Università di Firenze, Italia

Università di Roma La Sapienza, Italia

MEMOCS (ISSN 2325-3444 electronic, 2326-7186 printed) is a journal of the International Research Center for the Mathematics and Mechanics of Complex Systems at the Università dell'Aquila, Italy.

Cover image: "Tangle” by $\odot$ John Horigan; produced using the Context Free program (contextfreeart.org).

PUBLISHED BY

7 mathematical sciences publishers

nonprofit scientific publishing

http://msp.org/

(C) 2016 Mathematical Sciences Publishers 
Special issue in honor of

Lucio Russo

Lucio Russo: A multifaceted life

Raffaele Esposito and Francesco dell'Isola

The work of Lucio Russo on percolation Geoffrey R. Grimmett

"Mathematics" and "physics" in the science of harmonics Stefano Isola

From quantum to classical world: emergence of trajectories in a quantum system Rodolfo Figari and Alessandro Teta

Propagation of chaos and effective equations in kinetic theory: a brief survey Mario Pulvirenti and Sergio Simonella

What decides the direction of a current? Christian Maes

A remark on eigenvalue perturbation theory at vanishing isolation distance Fiorella Barone and Sandro Graffi

Some results on the asymptotic behavior of finite connection probabilities in percolation Massimo Campanino and Michele Gianfelice

Correlation inequalities for the Potts model Geoffrey R. Grimmett

Quantum mechanics: some basic techniques for some basic models, I: The models Vincenzo Grecchi

Quantum mechanics: some basic techniques for some basic models, II: The techniques Vincenzo Grecchi

On stochastic distributions and currents

Vincenzo Capasso and Franco Flandoli

A note on Gibbs and Markov random fields with constraints and their moments Alberto Gandolfi and Pietro Lenarda

Quantum mechanics: light and shadows (ontological problems and epistemic solutions) Gianfausto Dell'Antonio

MEMOCS is a journal of the International Research Center for the Mathematics and Mechanics of Complex Systems at the Università dell' Aquila, Italy.

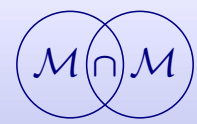

